\title{
A Study of Fasting Type 2 Diabetes Mellitus Patients With Acute Complications During Ramadan in Hospital Tengku Ampuan Afzan, Kuantan, Pahang
}

Nur Diyana Asyiqin Mansor ${ }^{1}$, Nur Faqihah Jaafar ${ }^{1}$, Abdullah Ammar Mohamed Ruslan ${ }^{1}$, Miza Hiryanti Zakaria ${ }^{2}$, Mohammad Arif Shahar ${ }^{1}$

${ }^{1}$ Department of Internal Medicine, Kulliyyah of Medicine, International Islamic University Malaysia

${ }^{2}$ Department of Internal Medicine, Hospital Tengku Ampuan Afzan, Kuantan

Presenter: Nur Diyana Asyiqin Mansor

Introduction: A large number of Muslims fast during the holy month of Ramadan worldwide. A specific approach is needed to manage diabetic patients who intend to fast during Ramadan because of potential harm. Thus, the study aimed to describe the sociodemography and the characteristic of fasting diabetic patients admitted due to acute diabetic complications during Ramadan. Materials and Methods: A cross-sectional study was conducted among fasting diabetic patients in Hospital Tengku Ampuan Afzan (HTAA) who were admitted due to severe hypoglycemia, diabetic ketoacidosis, hyperglycemic hyperosmolar syndrome and uncontrolled diabetes mellitus during the month of Ramadan $2017 / 1438 \mathrm{H}$. Subjects were interviewed and blood investigation results were obtained from medical records and recorded in patients' case report. Results: Thirteen patients who were admitted due to acute complications of diabetics during Ramadan were included in this study. Of these, $38.5 \%$ had severe hypoglycemia, $23.1 \%$ diabetic ketoacidosis, $15.4 \%$ hyperglycemia hyperosmolar syndrome and $23.1 \%$ uncontrolled diabetes mellitus. All of the patients had a long standing diabetes mellitus (median duration of 10 years). $\mathrm{HbA}_{1} \mathrm{C}$ was high (11.5\%) among hyperglycemic subjects. Types of treatment and previous history of severe hypoglycemia prior to Ramadan were the only independent factors identified to be related with acute complications of diabetes during fasting month $(p=0.030$ and $p=0.032$ respectively). Insulin, either alone or in combination with oral hypoglycemic agent, was associated with higher number of hypoglycemic event. Conclusion: The significant risk factors found were consistent with other studies. A thorough, individualized and holistic approach is needed in managing patients with diabetes who intend to fast. 